Original Paper http://ajol.info/index.php/ijbcs http://indexmedicus.afro.who.int

\title{
Réponse morphophysiologique du gombo [Abelmoschus esculentus (L.) Moench] soumis à la biofertilisation et à des stress hydriques
}

\author{
Bibata KONATE ${ }^{1 *}$, Rasmata NANA ${ }^{1}$, Sékeyoba Léopold NANEMA ${ }^{1}$, \\ Badoua BADIEL ${ }^{1}$, Mahamadou SAWADOGO ${ }^{2}$ et Zoumbiéssé TAMINI ${ }^{1}$ \\ ${ }^{I}$ Laboratoire Biosciences, Equipe d'Ecophysiologie Végétale, Université Ouagal Prof Joseph KI-ZERBO, 03 \\ BP 7021 Ouagadougou 03, Burkina Faso. \\ ${ }^{2}$ Laboratoire Biosciences, Equipe de Génétique et Amélioration des Plantes, Université Ouagal Prof Joseph \\ KI-ZERBO, 03 BP 7021 Ouagadougou 03, Burkina Faso. \\ "Auteur correspondant ; E-mail : bibatakonate36@gmail.com ; Tel : +226 78398566/ +226 71992987.
}

\section{RESUME}

La production agricole est non seulement influencée par le déficit pluviométrique mais aussi par la pauvreté des sols. L'objectif de cette étude est de connaître l'impact du fumier organique sur la réponse du gombo au déficit hydrique. Trois types de substrats ont été mis en place: Ft (100\% de terre), F1 (95\% de terre + $5 \%$ de fumier), F2 (90\% de terre $+10 \%$ de fumier). Le stress hydrique a été imposé au stade végétatif à partir

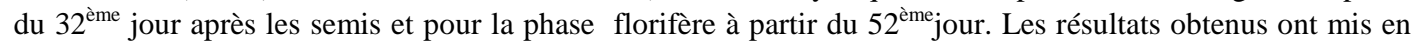
évidence l'augmentation de la teneur en eau du sol, ainsi que la teneur relative en eau des feuilles, avec l'augmentation des différentes doses de fumier chez toutes les plantes. Par contre, on note une diminution de la teneur en protéines, une réduction de la taille, du diamètre au collet, de la biomasse sèche totale et du nombre de capsules par plante chez les plantes stressées. Les plantes du substrat F1 enregistrent les meilleurs résultats aussi bien chez les plantes stressées que chez les plantes témoins :F1 est donc mieux adapté pour une culture de gombo dans des conditions hydriques déficitaires.

(C) 2016 International Formulae Group. All rights reserved.

Mots clés : Gombo, fumier organique, rendement.

\section{Morpho physiologicalresponse okra [Abelmoschus esculentus (L.) Moench] subject to biofertilization and water stress}

\begin{abstract}
Agricol production is not only influenced by pluviometric deficit but also by soil unfertility. Objective of this study is to know impact of organic fertilizer on okra response to hydric deficit. Three types of substrates were introduced: Ft (100\% of soil), F1 (95\% soil + 5\% manure), F2 (90\% soil + 10\% manure). Water stress was imposed at the vegetative stage from the 32th day after sowing and for the flowering phase from the 52nd day. The results obtained allowed to demonstrate an increase in the water content of the soil and the relative water content of leaves, with increasing manure different doses in all plants. By cons, there is a decrease in protein content, a reduction in size, the collar diameter, total dry biomass and the number of capsules per plant
\end{abstract}


in stressed plants. Plants of the F1 substrate post the best results both in stressed plants than in control plants : $\mathrm{F} 1$ as the dosage that can mitigate the effect of water stress in okra.

(C) 2016 International Formulae Group. All rights reserved.

Keywords: Okra, organic fertilizer, yield.

\section{INTRODUCTION}

L'agriculture des pays d'Afrique tropicale est confrontée à divers problèmes, tels que l'importante baisse de la pluviométrie, l'augmentation de sa variabilité spatio-temporaire et la péjoration climatique qui frappe l'ensemble des régions soudanosahéliennes depuis trois décennies (Koulibaly et al., 2002).

En plus des conditions pluviométriques drastiques, le Burkina Faso, à l'instar des pays Soudano-sahéliens, est soumis à une forte dégradation de ses terres cultivables provoquant leur appauvrissement. Il est donc difficile d'envisager une amélioration de la productivité agricole sans une restauration de la fertilité des sols naturellement faible. Les biofertilisants (fumure organique, compost ...) sont sources d'éléments fertilisants nécessaires aux plantes.

Jadis considéré comme une culture marginale (Sawadogo et al., 2009), le gombo constitue de nos jours un légume très rémunérateur pour les communautés pauvres $\mathrm{du}$ fait de son fort potentiel de vente sur les marchés ruraux et urbains. Il procure des revenus susceptibles de satisfaire de nombreux besoins tant industriels, familiaux que collectifs. Le gombo frais découpé séché et/ou transformé en poudre, est acheminé vers le marché extérieur, l'Europe et la sous région principalement (AADI, 2006). Le gombo possède également des vertus thérapeutiques diverses (Oyen et Jemmens, 2002). Les travaux de recherche réalisés en Chine ont révélé qu'un extrait alcoolique de feuilles de gombo est susceptible d'éliminer les radicaux libres de l'oxygène; réduire les maladies de l'insuffisance rénale, d'améliorer les fonctions rénales et de réduire la protéinurie (Siemonsma et Kouamé, 2004).
Vu toutes ces potentialités que possède le gombo, il est plus que nécessaire d'envisager des stratégies pour améliorer sa productivité afin d'augmenter son rendement, même sous l'influence des contraintes hydriques.

Le présent travail a pour objectif d'apprécier l'impact des différentes doses de fumier organique (fumier ovin) sur les réponses physiologiques, biochimiques et agronomiques d'une variété (UAE22) de gombo vis-à-vis du déficit hydrique.

\section{MATERIEL ET METHODES \\ Matériel végétal}

L'étude a porté sur une variété de gombo : UAE 22. Elle est l'une des 5 variétés obtenues par sélection variétale participative en 2005 par l'équipe d'amélioration génétique de gombo de l'UFR/SVT de l'Université de Ouagadougou. Le choix de cette variété a été effectué d'abord sur la base du fait qu'elle soit une variété locale et en plus du fait qu'elle a été identifiée comme la plus résistante au manque d'eau et la plus productive (Nana, 2010).

\section{Méthodes de culture}

Les plantes ont été cultivées dans des pots en plastique de forme conique, de diamètre égal à $33 \mathrm{~cm}$, d'une hauteur de 35 $\mathrm{cm}$, de capacité de 17 litres et dont le fond est troué pour éviter l'asphyxie racinaire. Chaque pot contenait 15 kilogrammes de substrats de culture. Trois types de substrat ont été utilisés: Ft $(100 \%$ de terre $)=$ substrat sans fumier ; $\mathrm{F} 1=95 \%$ de terre $+5 \%$ de fumier ; $\mathrm{F} 2=90 \%$ de terre $+10 \%$ de fumier (Tableau 1). Tous les pots ont reçu cinq (5) graines semées à une profondeur de $5 \mathrm{~cm}$. Un démariage à un plant par pot a été effectué le $14^{\text {ème }}$ jour après les semis. 


\section{Dispositif expérimental}

L'expérimentation a été réalisée suivant un dispositif «split-plot» randomisé à trois répétitions. L'essai a compté au total 81 pots ( 3 variantes de substrat $\mathrm{x} 3$ pots /variante $\mathrm{x} 3$ variantes de traitement hydrique $\mathrm{x} 3$ répétitions). Les traitements représentent les sous blocs et les pots correspondent à l'unité expérimentale. Les blocs constituent les répétitions. Le traitement $\mathrm{T} 0$ représente le lot de plantes témoins sans restriction hydrique arrosé chaque deux jours jusqu'à la fin du cycle, T1 représente le lot de plantes stressées au stade végétatif c'est-à-dire avant l'apparition des boutons floraux et $\mathrm{T} 2$ est le lot de plantes stressées au stade florifère (Figure 1).

\section{Traitements hydriques}

Les plantes ont été soumises à trois niveaux d'alimentations hydriques au cours de l'expérimentation :

- Arrosage régulier chaque deux jours à la capacité au champ (CAC) du sol jusqu'à la fin de l'expérimentation (T0).

- Arrosage chaque deux jours à la capacité au champ (CAC) du sol avec un arrêt pendant 15 jours au stade végétatif (T1). Le traitement (T1) n'a été appliqué que lorsque les feuilles de rang quatre (à partir de l'apex) étaient épanouies pour permettre les mesures et les échantillonnages.

- Arrosage chaque deux jours à la capacité au champ (CAC) du sol avec une interruption à la floraison (T2). Le traitement (T2) n'a été appliqué que lorsqu'on avait au moins 50\% de plantes qui ont eu une fleur ouverte.

Les prélèvements des échantillons de feuilles ont été faits le dernier jour de stress sur la $4{ }^{\text {ème }}$ feuille apicale de chaque plant pour l'évaluation du statut hydrique et sur la $3^{\text {ème }}$ feuille apicale stockée dans de l'azote liquide à $195{ }^{\circ} \mathrm{C}$ pour la mesure des autres paramètres.

\section{Méthodes de mesure}

\section{Détermination de la teneur en eau du sol $\left(\boldsymbol{T E} E_{\text {sol }}\right)$}

Au dernier jour de stress hydrique, des échantillons de sol ont été prélevés dans les pots de chaque traitement hydrique, à une profondeur de $20 \mathrm{~cm}$ avec des boîtes en aluminium et pesés pour obtenir le poids frais (PF). Les échantillons de sol ont été ensuite séchés à l'étuve à $105{ }^{\circ} \mathrm{C}$ pendant 48 heures et la teneur en eau du sol $\left(\mathrm{TE}_{\mathrm{SOL}}\right)$ a été déterminée comme suit:

$$
\mathrm{TE}_{\mathrm{SOL}}(\%)=\frac{\mathrm{PF}-\mathrm{PS}}{\mathrm{PS}} \times 100
$$

Mesure de l'état hydrique des plantes: teneur relative en eau (TRE) des feuilles

$\mathrm{La}$ mesure de la turgescence relative est effectuée selon la méthode décrite par Barrs (1968). La quatrième feuille à partir de l'apex de chaque plant, coupée à la base du limbe est pesée immédiatement pour avoir le poids frais (PF). La saturation est obtenue en plaçant la feuille entière dans un flacon contenant $200 \mathrm{ml}$ d'eau distillée et laissé à l'obscurité à une température de $4{ }^{\circ} \mathrm{C}$ pendant 24 heures, puis la feuille a été pesée à nouveau pour obtenir le poids de la pleine turgescence (PPT), le poids sec est obtenu par dessiccation des feuilles à l'étuve réglée à 80 ${ }^{\circ} \mathrm{C}$ pendant 24 heures. La teneur relative en eau (TRE) est calculée suivant la formule:

$$
\operatorname{TRE}(\%)=\frac{\mathrm{PF}-\mathrm{PS}}{\mathrm{PPT}-\mathrm{PS}} \times 100
$$

\section{Dosage des protéines totales}

Le dosage a été fait selon la méthode décrite par Bradfort(1976).

\section{Préparation du Bleu de Coomassie}

La solution de CBBG-250 (Coomassie Brillant Bleu G-250) a été préparée à une concentration de $0,06 \%(\mathrm{P} / \mathrm{V})$ dans une solution d'acide perchlorique à $3 \%(\mathrm{P} / \mathrm{V})$. La solution est laissée sous agitation pendant 24 heures puis filtrée. La solution est ajustée pour 
donner une densité optique (DO) comprise entre 1,3 et 1,5 à $450 \mathrm{~nm}$, à l'aide d'une solution d'acide perchlorique.

Préparation de la courbe d'étalonnage pour le dosage des protéines

La protéine standard utilisée pour le dosage des protéines a été le Bovin Serum Albumin (BSA) à une concentration de 50 $\mu \mathrm{g} / \mathrm{ml}$ dans l'eau distillée.

La préparation de l'étalon des protéines se fait suivant le Tableau 2.

\section{Solution d'extraction des protéines de l'échantillon}

Elle été obtenue en mélangeant 7,5 g de Sodium Dodecy Sulfate (SDS)à $225 \mu \mathrm{de}$ $\beta$-mercapto-éthanol et le tout a été complété à $500 \mathrm{ml}$ avec l'eau distillée.

\section{Dosage de l'échantillon}

La solution d'échantillon a été obtenue par dissolution de $5 \mathrm{~g}$ de matériel végétal biologique dans $12,5 \mathrm{ml}$ de la solution d'extraction. Cette solution est ensuite centrifugée à 5000 tours $/ \mathrm{mn}$ pendant $5 \mathrm{mn}$. Après centrifugation, le surnageant est recueilli, filtré avec un papier filtre, juste pour retenir les particules solides puis dilué 10 fois. Le dosage s'effectue selon le Tableau 3.

\section{Mesures de la croissance verticale et radiale des plantes}

Les mesures de la croissance des plants

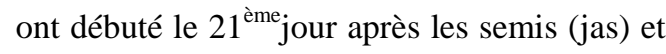
ont été effectuées régulièrement chaque 7 jour jusqu'à la fin des expérimentations. Les mesures de la croissance verticale et de celle radiale consistent respectivement en la mesure de la hauteur et du diamètre au collet au fil du temps. La hauteur de chaque plant (HP) exprimée en centimètres $(\mathrm{cm})$ est mesurée à l'aide d'un ruban et le diamètre au collet (DC) de la tige, exprimé en millimètres $(\mathrm{mm})$ est mesuré à l'aide d'un pied à coulisse.

\section{Paramètres agronomiques}

Les paramètres agronomiques ont concerné le nombre de capsules par plante, le poids sec moyen d'une capsule (g), le poids $\mathrm{sec}$ de la partie aérienne végétative $(\mathrm{g})$, le poids sec de la partie racinaire $(\mathrm{g})$, la biomasse sèche totale $(\mathrm{g})$.

Le rendement en capsules a été estimé à la suite d'une cueillette progressive chaque trois jour. Les capsules cueillies sont découpées en rondelle, séchées naturellement au soleil pour évaluer le poids sec. Le poids sec de la partie aérienne végétative et celui de la partie racinaire ont été déterminés après passage à l'étuve à $80{ }^{\circ} \mathrm{C}$ pendant $72 \mathrm{~h}$ à l'aide d'une balance de précision $0,01 \mathrm{~g}$. Le rapport du rendement en capsules par la biomasse totale donne l'indice de récolte ou Harvest index (HI).

\section{Traitement et analyse statistiques des données}

Les données ont été calculées sous forme d'une moyenne de 3 répétitions à l'aide du tableur EXCEL 2007 et les graphiques également réalisés à l'aide du même tableur. Pour apprécier les différences entre les variantes des substrats de culture, les traitements hydriques et identifier les corrélations entre les paramètres, les données obtenues ont fait l'objet d'une analyse de la variance (ANOVA) et d'une analyse en composante principale (ACP). Les tests de comparaison des moyennes sont effectués selon la méthode de Newman-Keuls. Les niveaux de signification des traitements des substrats de culture et des interactions traitement hydrique $\mathrm{x}$ substrats sont déterminés à un niveau de probabilité de 0,05. Toutes ces analyses ont été réalisées avec le logiciel XLSTAT Pro.

\section{RESULTATS \\ Teneur en eau du sol}

La teneur en eau du sol est représentative de l'état hydrique moyen du sol perçu par les racines. La teneur en eau moyenne du sol évaluée à la fin de chaque phase de stress hydrique, fait ressortir une baisse chez les plantes stressées par rapport à celle des témoins. Cette réduction est en 
moyenne de $14,97 \%$ au stade végétatif et $15,04 \%$ au stade floraison. D'autre part au sein de chaque traitement (végétatif et floraison), la teneur en eau élevée du sol s'observe au niveau des substrats de culture où il $\mathrm{y}$ a plus de fumier (F2) ensuite en F1 et enfin au niveau du substrat $(\mathrm{Ft})$ où il n'y a pas de fumier. A titre indicatif, on a au stade végétatif, $15,28 \%$ d'eau chez les plantes témoins contre $4,15 \%$ chez les plantes stressées, pour le substrat de culture $(\mathrm{Ft})$; $22,25 \%$ d'eau chez les plantes témoins contre $5,24 \%$ chez les plantes stressées, pour le substrat de culture contenant $5 \%$ de fumier (F1) et $23,79 \%$ d'eau chez les plantes témoins contre $7,01 \%$ chez les plantes stressées, pour le substrat de culture contenant $10 \%$ de fumier (F2). Au stade floraison, on a 14,25\% d'eau chez les plantes témoins contre $2,31 \%$ chez les plantes stressées, pour le substrat Ft; $21,04 \%$ d'eau chez les plantes témoins contre $4,18 \%$ chez les plantes stressées, pour le substrat $\mathrm{F} 1$ et $22,15 \%$ d'eau chez les plantes témoins contre $5,82 \%$ chez les plantes stressées, pour le substrat F2 (Figure 2). Il ressort que le substrat de culture F2 est celui qui a la plus forte teneur en eau aussi bien au stade végétatif qu'au stade floraison.

Etat hydrique des plantes: la teneur relative en eau (TRE) des feuilles

La teneur relative en eau (TRE) est l'un des paramètres les plus utilisés et les plus importants pour évaluer le statut hydrique de la plante. Au stade végétatif comme au stade floraison, la TRE des feuilles des plantes stressées a diminué par rapport à celle des plantes témoins (Figure 3). La baisse remarquable de la TRE au stade floraison, suppose que l'impact du déficit hydrique sur la plante est fonction de son degré de développement. Ainsi, au stade végétatif de la variété UAE22 l'effet du déficit hydrique sur la TRE, a été moindre comparativement à son effet au stade floraison. Cependant, comme la teneur en eau du sol, la TRE des feuilles est plus élevée dans les feuilles des plantes cultivées sur le substrat de culture F2 au cours des deux stades de développement (végétatif et floraison). Le stress intervenu au stade végétatif révèle pour les trois substrats de culture Ft, F1, F2, respectivement une TRE de $85,95 \% ; 86,65 \% ; 90,79 \%$ pour les plantes témoins et $58,05 \% ; 60,48 \% ; 62,5 \%$ pour les plantes stressées (Figure 3A). Par contre, lorsque le stress hydrique a été induit à la floraison, les TRE sont plus faibles avec respectivement $78,62 \% ; 80,5 \%$ et $84,10 \%$ pour les plantes témoins et $43,63 \% ; 55,55 \%$; $58,68 \%$ pour les plantes stressées (Figure 3B).

Le test de Newman et Keuls au seuil de $5 \%$ ne fait ressortir aucune différence significative entre l'influence des différents substrats de culture sur le déficit hydrique appliqué au stade floraison (Tableau 4) où $\mathrm{P}=$ 0,117. Cependant, au stade végétatif, une différence significative s'est dégagée $(\mathrm{P}=$ 0,030). En plus, l'effet du traitement hydrique est fortement significatif $(\mathrm{P}<0,0001)$ au stade végétatif ainsi qu'au stade floraison.

L'analyse du Tableau 4 fait donc ressortir des interactions «traitements hydriques $\mathrm{x}$ substrats de culture » significatives chez les plantes stressées au stade végétatif et permet de distinguer deux (2) groupes tandis que les plantes témoins en forment un (1) seul groupe. La conséquence est que les plantes stressées cultivées sur le substrat de culture Ft et F1 forment un groupe homogène qui présente une TRE plus faible par rapport à celles cultivées sur le substrat F2 qui forment un autre groupe homogène. Cependant, au stade floraison, l'interaction «traitements hydriques x substrats de culture » est non significative chez les plantes témoins et chez les plantes stressées si bien que toutes les interactions forment un seul groupe statistique avec une TRE plus importante en F2 dans tous les cas.

\section{La croissance verticale}

Le stress hydrique induit au stade végétatif ainsi qu'au stade floraison, a eu un effet dépressif sur la croissance verticale des plantes cultivées par rapport aux plantes témoins dans toutes les trois variantes de 
substrat de culture (Ft, F1, F2). Cette dépression est faible chez les plantes stressées en période de floraison (Figure 4). C'est ainsi qu'on a en moyenne $16,69 \%$ de réduction pour les plantes stressées au stade végétatif et $5,13 \%$ pour celles stressées au stade floraison. Des trois substrats de culture, c'est sur le substrat F1 que les plantes ont présenté les hauteurs finales importantes, ensuite sur F2 et enfin sur le substrat témoin.

L'analyse de la variance ne révèle aucune différence significative des interactions entre le régime hydrique et le type de substrat.

\section{La croissance radiale}

Le même phénomène observé au niveau de la croissance verticale est également constaté au niveau de la croissance radiale à savoir les résultats similaires. La croissance verticale est négativement affectée par le stress hydrique intervenu au stade végétatif avec des diamètres au collet élevés obtenus avec le substrat F1 (Figure 5). L'analyse de la variance ne fait pas ressortir de différence d'interaction des substrats de culture sur les traitements hydriques. Toutefois, il y a un écart de taille entre les plantes témoins et les plantes stressées au stade végétatif comme celles stressées au stade floraison.

\section{La teneur en protéines totales}

L'évolution des teneurs en protéines totales, extraites à partir des feuilles fraîches de la variété UAE 22 de gombo fait ressortir une baisse chez toutes les plantes stressées aussi bien au stade végétatif qu'au stade floraison comparativement à celles des témoins (Figure 6). Cette baisse est relativement plus importante à la floraison, soit $32,07 \%$ contre $28,79 \%$ au stade végétatif. L'analyse de la variance ne fait pas ressortir de différence d'interaction des substrats de culture sur les traitements hydriques. Cependant, les plus teneurs faibles en protéines s'observent chez les plantes cultivées sur le substrat F2.

\section{Les paramètres agronomiques}

Le Tableau 5 présente les différents paramètres agronomiques étudiés à savoir le nombre de capsules par plante, le poids sec moyen d'une capsule, le poids sec de la partie aérienne, le poids sec de la partie végétative, le poids sec de la partie racinaire, la biomasse sèche totale et l'indice de Havers. L'analyse de ce tableau montre que chez les plantes du substrat de culture sans fumier(Ft), le stress hydrique intervenu durant les deux stades de développement a eu un effet positif sur la production des capsules caractérisé par une hausse de celles-ci. Avec les substrats F1 et $\mathrm{F} 2$, au stade végétatif il y a eu une réduction du rendement en capsules et au stade floraison une hausse avec le substrat F2 tandis qu'on observe une diminution du nombre de capsules avec le substrat F1. Quant au poids sec d'une capsule, il est inversement proportionnel au nombre de capsules par plante chez les plantes qui se sont développées sur le substrat sans fumier Ft indépendamment du traitement hydrique. On pourrait insinuer qu'une faible production en capsules (Tableau 5) favorise l'augmentation de leur taille et par conséquent le poids sec de la capsule. Cependant, sous l'influence des substrats F1 et $\mathrm{F} 2$ c'est le phénomène contraire qui est constaté.

D'une manière générale, le stress hydrique appliqué au stade végétatif comme à la floraison a eu une influence négative sur la biomasse sèche totale de toutes les plantes stressées sauf chez celles du substrat sans fumier $\mathrm{Ft}$ où il y a eu une hausse. Cette biomasse sèche totale étant le cumul de la partie sèche végétative et de la partie sèche racinaire, ces dernières subissent la même hausse ou la même réduction. Ainsi, par rapport aux plantes témoins on a une hausse de $12,99 \%$ au stade végétatif $\mathrm{T} 1,3,08 \%$ au stade floraison $\mathrm{T} 2$ avec le substrat de culture $\mathrm{Ft}$; une réduction de $29,42 \%$ en $\mathrm{T} 1,29,33 \%$ en $\mathrm{T} 2$ avec le substrat $\mathrm{F} 1$; une réduction de $8,49 \%$ en $\mathrm{T} 1 ; 12,85 \%$ en $\mathrm{T} 2$ avec le substrat F2. Par ailleurs, les plantes cultivées sur le substrat sans fumier $\mathrm{Ft}$ ont un indice de récolte le plus élevé soit en moyenne 0,51; 
0,43 pour celles du substrat $\mathrm{F} 1$ et 0,33 pour celles du substrat F2.

L'analyse de variance n'a décelé aucune différence significative des interactions « substrats de culture $\mathrm{x}$ traitements hydriques». Cependant, en dehors de l'indice de récolte les plantes qui ont évolué sur le substrat F1 ont les meilleurs résultats pour les paramètres agronomiques.

Relation entre la croissance et les paramètres agronomiques

$\mathrm{Au}$ regard de la matrice de corrélation de Pearson, chez les plantes témoins non stressées (Tableau 6A), des corrélations positives et très significatives sont observées entre tous les paramètres de croissance et agronomiques à l'exception de l'indice de récolte.

Cependant, chez les plantes stressées au stade végétatif (Tableau 6B), les corrélations positives qui existaient à la fois entre la hauteur, le poids sec racinaire et le poids sec moyen d'une capsule; entre le diamètre et le poids sec racinaire ; ainsi qu'entre le poids sec racinaire et le poids sec moyen d'une capsule n'y sont plus observées.

Par contre, lorsque le stress hydrique a été appliqué à la floraison (Tableau 6C), ce sont les corrélations entre la hauteur et le diamètre, entre la hauteur et le poids sec racinaire, entre le diamètre et le nombre de capsules par plante qui ne sont pas maintenues.

Tableau 1:Caractéristiques physico-chimiques des trois variantes de substrats de culture.

\begin{tabular}{|c|c|c|c|c|c|c|c|c|c|c|}
\hline $\begin{array}{l}\text { Substrats } \\
\text { de } \\
\text { culture }\end{array}$ & $\begin{array}{l}\text { Argiles } \\
(\%)\end{array}$ & $\begin{array}{l}\text { Limons } \\
(\%)\end{array}$ & $\begin{array}{l}\text { Sables } \\
(\%)\end{array}$ & $\begin{array}{l}\text { MOT } \\
(\%)\end{array}$ & $\begin{array}{l}\mathrm{C} \\
(\%)\end{array}$ & $\begin{array}{l}\mathrm{N} \\
(\%)\end{array}$ & $\mathrm{C} / \mathrm{N}$ & $\begin{array}{l}\mathbf{P} \\
(\mathbf{p p m})\end{array}$ & $\begin{array}{l}\text { K } \\
(\mathbf{p p m})\end{array}$ & $\begin{array}{l}\text { pH eau } \\
(\mathbf{p} / \mathbf{v}: \\
2 / 5)\end{array}$ \\
\hline Ft & 21,57 & 17,65 & 60,78 & 0,802 & 0,465 & 0,037 & 12,56 & 884,6 & 924,8 & 6,17 \\
\hline F1 & 20,50 & 16,76 & 57,74 & 2,552 & 1,480 & 0,134 & 11,04 & 1280,3 & 2425,1 & 7,91 \\
\hline F2 & 19,41 & 15,88 & 54,70 & 3,322 & 1,927 & 0,168 & 11,47 & 1676 & 3146,1 & 8,38 \\
\hline
\end{tabular}

Tableau 2 : Préparation de l'étalon des protéines.

\begin{tabular}{lllll}
\hline \multicolumn{1}{c}{ Tube } & T0 (Blanc) & T1 & T2 & T3 \\
\hline BSA (50 $\boldsymbol{\mu g} / \mathbf{m l})$ en $\mathbf{~ m l}$ & 0 & 0,1 & 0,2 & 0,3 \\
Eau distillée $(\mathbf{m l})$ & 1 & 0,9 & 0,8 & 0,7 \\
Réactif Bleu de Coomassie $(\mathbf{m l})$ & 1 & 1 & 1 & 1
\end{tabular}

Après avoir ajouté le réactif, les densités optiques sont lues dans les 2 à 5 minutes qui suivent à $620 \mathrm{~nm}$ et $450 \mathrm{~nm}$. T1 = plantes témoins ; $\mathbf{T} \mathbf{2}=$ plantes stressées au stade végétatif ; $\mathbf{T 3}=$ plantes stressées au stade floraison ; $\mathrm{BSA}=$ Bovin Serum Albumin.

Tableau 3 : Dosage d'échantillons des protéines.

\begin{tabular}{lllll}
\hline Tube & T0 (Blanc) & T1 & T2 & T3 \\
\hline Echantillon & 0 & 0,1 & 0,1 & 0,1 \\
Solution d'extraction en $\mathbf{~ m l}$ & 0,01 & 0 & 0 & 0 \\
Eau distillée en $\mathbf{m l}$ & 0,990 & 0,9 & 0,9 & 0,9 \\
Réactif Bleu de Coomassie $(\mathbf{m l})$ & 1 & 1 & 1 & 1 \\
\hline
\end{tabular}


Tableau 4 : Analyse des interactions «traitements hydriques x substrats de culture» sur la teneur relative en eau.

\begin{tabular}{llcll}
\hline Substrats de & \multicolumn{3}{c}{ Stade végétatif } & \\
\cline { 2 - 5 } culture & T0 & T1 & T0 & T2 \\
Ft & $85,613^{\mathrm{a}}$ & $57,526^{\mathrm{b}}$ & $80,80^{\mathrm{ab}}$ & $44,992^{\mathrm{c}}$ \\
F1 & $86,393^{\mathrm{a}}$ & $59,410^{\mathrm{b}}$ & $80,605^{\mathrm{ab}}$ & $50,615^{\mathrm{c}}$ \\
F2 & $89,090^{\mathrm{a}}$ & $63,124^{\mathrm{a}}$ & $80,636^{\mathrm{ab}}$ & $57,719^{\mathrm{c}}$ \\
\hline
\end{tabular}

Les moyennes d'un même traitement suivies de la même lettre ne sont pas significativement différentes au seuil de $5 \%$. T0 = plantes témoins $; \mathbf{T 1}=$ plantes stressées au stade végétatif $; \mathbf{T} 2=$ plantes stressées au stade floraison $\mathbf{F t}=$ substrat de culture sans fumier $(100 \%$ de terre) $; \mathbf{F 1}=$ substrat de culture composé de $95 \%$ de terre $+5 \%$ de fumier $; \mathbf{F} 2=$ substrat de culture contenant $90 \%$ de terre $+10 \%$ de fumier.

Tableau 5: Rendement et ses composantes des plantes cultivées dans les trois milieux de culture.

\begin{tabular}{|c|c|c|c|c|c|c|c|}
\hline \multicolumn{2}{|c|}{$\begin{array}{l}\text { Caractères } \\
\text { Facteurs }\end{array}$} & NCap/pt & PS/Cap (g) & $\begin{array}{ll}\text { PSPA } & \text { (g) }\end{array}$ & PSR (g) & BST (g) & HI \\
\hline \multirow{3}{*}{ Ft } & T0 & 0,78 & 0,67 & $1,82 \pm 0,99$ & $0,39 \pm 0,22$ & $2,21 \pm 1,17$ & $0,55 \pm 0,35$ \\
\hline & T1 & 1 & 0,99 & $2,13 \pm 0,43$ & $0,41 \pm 0,10$ & $2,54 \pm 0,49$ & $0,42 \pm 0,10$ \\
\hline & $\mathbf{T} 2$ & 1,33 & 0,70 & $1,89 \pm 0,78$ & $0,39 \pm 0,13$ & $2,28 \pm 0,90$ & $0,58 \pm 0,28$ \\
\hline \multirow{3}{*}{ F1 } & T0 & 3,89 & 1,34 & $9,13 \pm 4,92$ & $1,88 \pm 1,67$ & $11,01 \pm 6,30$ & $0,40 \pm 0,12$ \\
\hline & T1 & 2,67 & 1,21 & $6,56 \pm 3,27$ & $1,22 \pm 0,76$ & $7,77 \pm 3,93$ & $0,50 \pm 0,29$ \\
\hline & $\mathbf{T} 2$ & 2,89 & 1,19 & $6,62 \pm 2,63$ & $1,16 \pm 0,44$ & $7,78 \pm 2,71$ & $0,39 \pm 0,14$ \\
\hline \multirow{3}{*}{ F2 } & T0 & 2,67 & 1,29 & $7,45 \pm 2,27$ & $1,03 \pm 0,30$ & $8,48 \pm 2,48$ & $0,31 \pm 0,10$ \\
\hline & T1 & 2 & 1,59 & $6,65 \pm 0,79$ & $1,10 \pm 0,79$ & $7,76 \pm 5,20$ & $0,29 \pm 1,67$ \\
\hline & T2 & 2,89 & 1,14 & $6,37 \pm 2,21$ & $1,02 \pm 0,33$ & $7,39 \pm 2,48$ & $0,40 \pm 0,15$ \\
\hline
\end{tabular}

$\mathbf{N C a p} / \mathbf{p t}=$ nombre de capsules par plante, $\mathbf{P S} / \mathbf{C a p}=$ poids sec par capsule, $\mathbf{P S P A}=$ poids sec partie aérienne, $\mathbf{P S R}=$ poids sec racinaire, BST = Biomasse sèche totale, $\mathbf{H I}=$ harvest index. $\mathbf{F t}=$ substrat de culture sans fumier $(100 \%$ de terre $), \mathbf{F 1}=$ substrat de culture composé de $95 \%$ de terre $+5 \%$ de fumier, $\mathbf{F} 2=$ substrat de culture contenant $90 \%$ de terre $+10 \%$ de fumier. $\mathbf{T} \mathbf{0}=$ plantes témoins, $\mathbf{T 1}=$ plantes stressées au stade végétatif, $\mathbf{T} \mathbf{2}$ = plantes stressées à la floraison.

Tableau 6:Matrice de corrélation entre les paramètres physiologiques (croissance) et agronomiques. Tableau 6A: Plantes témoins.

\begin{tabular}{lllllllll}
\hline & Hauteur & Diamètre & PSVég & PSRacin & BSTotale & NCap/plte & PSM/cap & HI \\
\hline Hauteur & & & & & & & & \\
Diamètre & $\mathbf{0 , 9 4 2}$ & & & & & & & \\
PSVég & $\mathbf{0 , 8 4 7}$ & $\mathbf{0 , 8 5 9}$ & & & & & & \\
PSRacin & $\mathbf{0 , 7 0 5}$ & $\mathbf{0 , 7 4 1}$ & $\mathbf{0 , 8 3 3}$ & & & & & \\
BSTotale & $\mathbf{0 , 8 4 4}$ & $\mathbf{0 , 8 6 0}$ & $\mathbf{0 , 9 9 7}$ & $\mathbf{0 , 8 7 6}$ & & & & \\
NCap/plte & $\mathbf{0 , 8 2 4}$ & $\mathbf{0 , 8 8 6}$ & $\mathbf{0 , 9 6 6}$ & $\mathbf{0 , 7 5 9}$ & $\mathbf{0 , 9 5 6}$ & & & \\
PSM/cap & $\mathbf{0 , 8 7 9}$ & $\mathbf{0 , 8 1 4}$ & $\mathbf{0 , 8 1 0}$ & $\mathbf{0 , 8 1 9}$ & $\mathbf{0 , 8 2 9}$ & $\mathbf{0 , 6 9 6}$ & & \\
HI & $-0,145$ & $-0,168$ & $-0,304$ & $-0,310$ & $-0,311$ & $-0,126$ & $-0,471$ & \\
\hline
\end{tabular}

PSVég $=$ partie sèche végétative, PSRacin $=$ partie sèche racinaire,BSTotale $=$ Biomasse sèche totale,NCap/pte $=$ nombre de capsules par plante, PSM/Cap = poids sec moyen par capsule, $\mathbf{H I}=$ harvest index. 
Tableau 6B : Plantes stressées au stade végétatif.

\begin{tabular}{|c|c|c|c|c|c|c|c|c|}
\hline & Hauteur & Diamètre & PSVég & PSRacin & BSTotale & NCap/plte & PSM/cap & HI \\
\hline \multicolumn{9}{|l|}{ Hauteur } \\
\hline Diamètre & 0,725 & & & & & & & \\
\hline PSVég & 0,792 & 0,774 & & & & & & \\
\hline PSRacin & 0,581 & 0,489 & 0,861 & & & & & \\
\hline BSTotale & 0,766 & 0,733 & 0,994 & 0,910 & & & & \\
\hline NCap/plte & 0,733 & 0,694 & 0,961 & 0,805 & 0,951 & & & \\
\hline PSM/cap & 0,441 & 0,789 & 0,737 & 0,448 & 0,694 & 0,799 & & \\
\hline HI & 0,284 & 0,271 & 0,091 & $-0,183$ & 0,037 & 0,287 & 0,477 & \\
\hline \multirow{2}{*}{\multicolumn{9}{|c|}{$\begin{array}{l}\text { PSVég = partie sèche végétative, } \text { PSRacin = partie sèche racina } \\
\text { de capsules par plante, PSM/Cap = poids sec moyen par capsule, } \\
\text { Tableau 6C : Plantes stressées au stade floraison. }\end{array}$}} \\
\hline & & & & & & & & \\
\hline & Hauteur & Diamètre & PSVég & PSRacin & BSTotale & NCap/plte & PSM/cap & HI \\
\hline \multicolumn{9}{|l|}{ Hauteur } \\
\hline Diamètre & 0,638 & & & & & & & \\
\hline PSVég & 0,761 & 0,784 & & & & & & \\
\hline PSRacin & 0,635 & $\mathbf{0 , 8 7 7}$ & 0,869 & & & & & \\
\hline BSTotale & 0,756 & $\mathbf{0 , 8 0 8}$ & 0,998 & 0,898 & & & & \\
\hline NCap/plte & 0,679 & 0,635 & 0,942 & 0,723 & 0,928 & & & \\
\hline PSM/cap & 0,686 & 0,964 & 0,875 & $\mathbf{0 , 9 2 7}$ & 0,895 & 0,710 & & \\
\hline HI & $-0,353$ & $-0,600$ & $-0,522$ & $-0,630$ & $-0,544$ & $-0,254$ & $-0,685$ & \\
\hline
\end{tabular}

PSVég $=$ partie sèche végétative, PSRacin $=$ partie sèche racinaire,BSTotale $=$ Biomasse sèche totale,NCap/pte $=$ nombre de capsules par plante, $\mathbf{P S M} / \mathbf{C a p}=$ poids sec moyen par capsule, $\mathbf{H I}=$ harvest index.

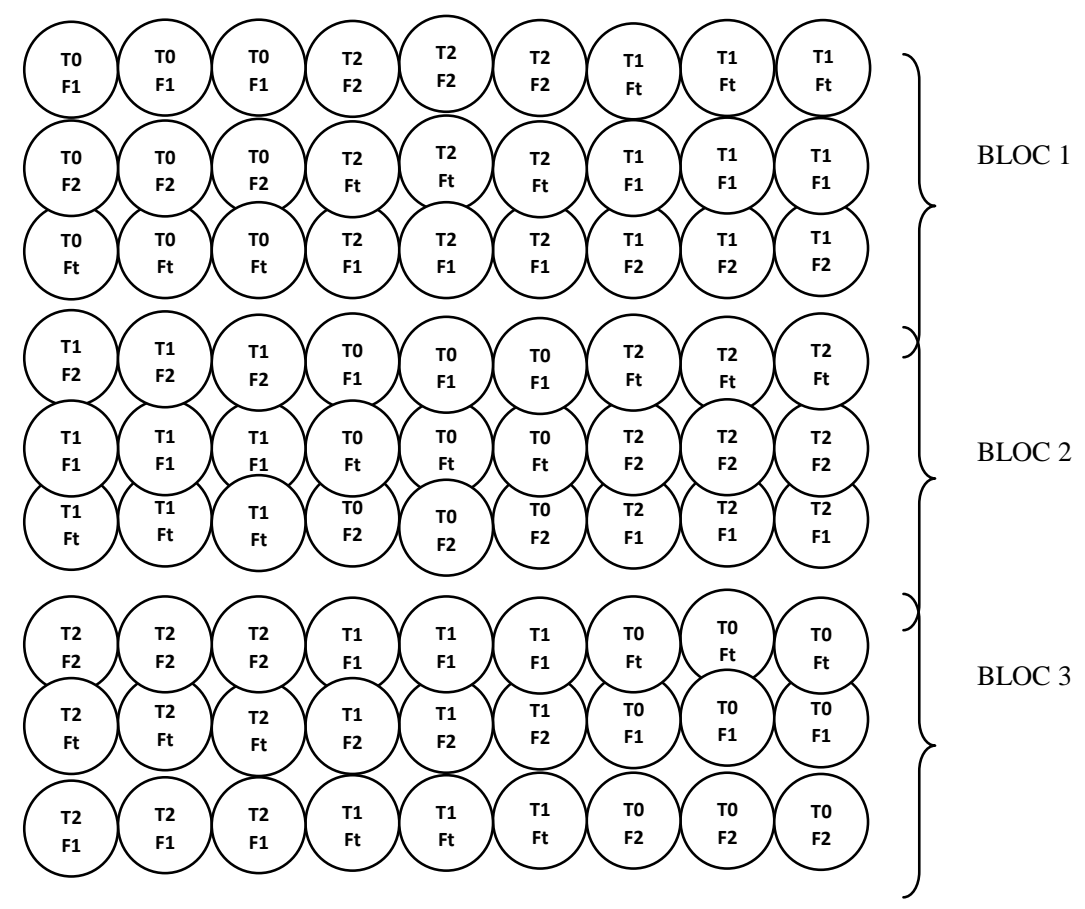

Figure 1: Dispositif expérimental mis en place. 

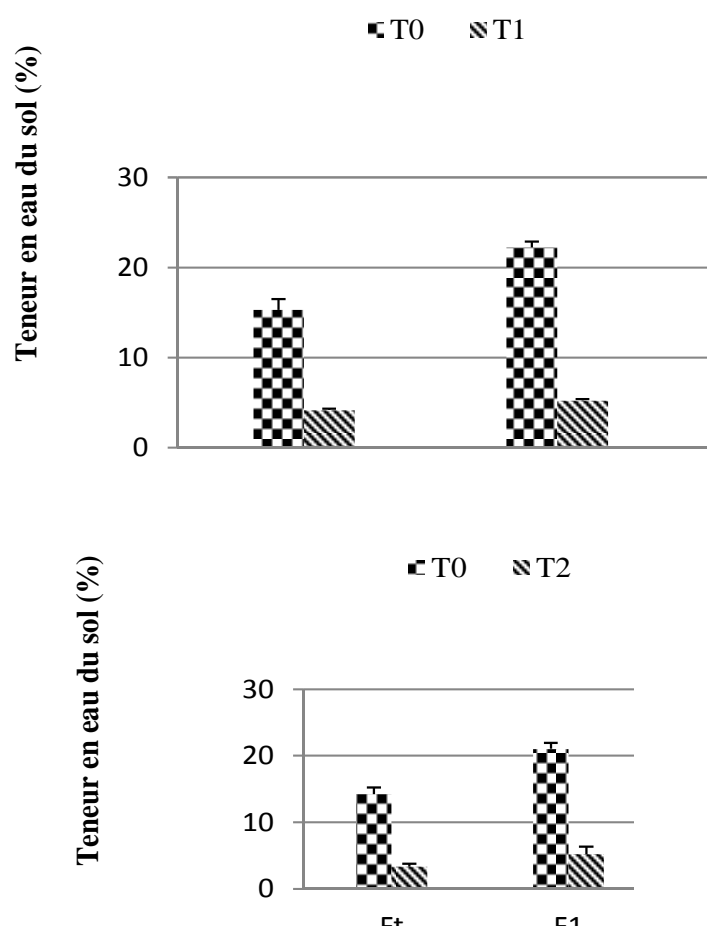

Figure 2: Variation de la teneur en eau du sol des trois substrats de culture chez les plantes d'UAE22 stressées au stade végétatif (A) et au stade floraison (B). T0 $=$ Plantes témoins ; $\mathbf{T 1}=$ Plantes stressées au stade végétatif $; \mathbf{T} 2=$ Plantes stressées au stade floraison $; \mathbf{F} \mathbf{t}=$ substrat de culture sans fumier $(100 \%$ de terre $) ; \mathbf{F} \mathbf{1}=$ substrat de culture composé de $95 \%$ de terre $+5 \%$ de fumier $; \mathbf{F} 2=$ substrat de culture contenant $90 \%$ de terre $+10 \%$ de fumier.

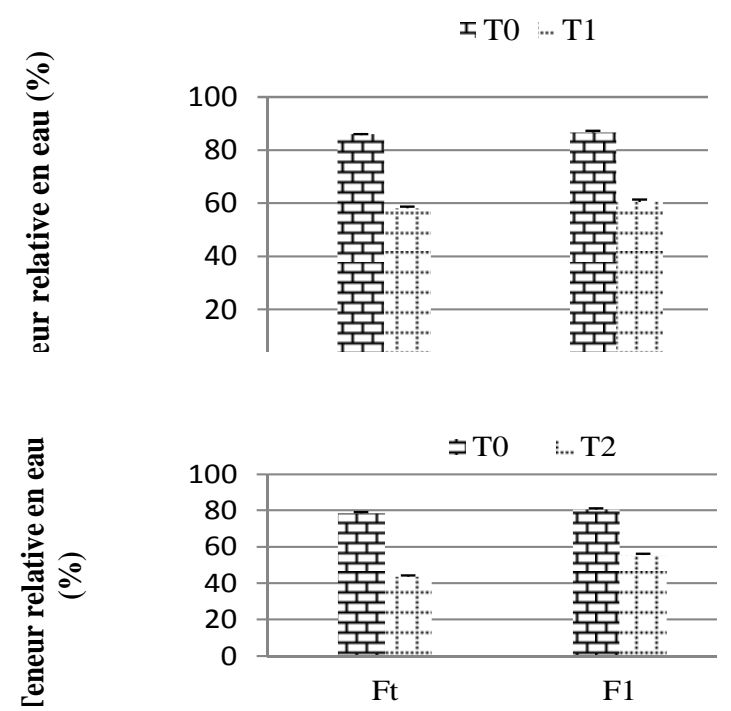

Figure 3 : Variation de la teneur relative en eau des feuilles des plantes d'UAE 22 stressées au stade végétatif (A), et au stade floraison (B) cultivées sur les trois substrats. $\mathbf{T} \mathbf{0}=$ plantes témoins; $\mathbf{T 1}=$ plantes stressées au stade végétatif $; \mathbf{T 2}=$ plantes stressées au stade floraison $; \mathbf{F t}=$ substrat de culture sans fumier $(100 \%$ de terre $) ; \mathbf{F 1}$ $=$ substrat de culture composé de $95 \%$ de terre $+5 \%$ de fumier $; \mathbf{F 2}=$ substrat de culture contenant $90 \%$ de terre $+10 \%$ de fumier. 

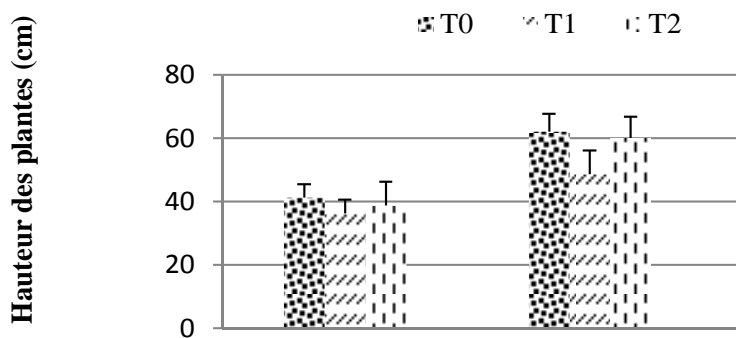

Figure 4: Hauteur finale $(\mathrm{cm})$ des plantes d'UAE 22 cultivées sur les trois substrats. To = plantes témoins $; \mathbf{T 1}=$ plantes stressées au stade végétatif $; \mathbf{T} 2=$ plantes stressées au stade floraison $; \mathbf{F t}=$ substrat de culture sans fumier $(100 \%$ de terre) $; \mathbf{F 1}=$ substrat de culture composé de $95 \%$ de terre $+5 \%$ de fumier $; \mathbf{F 2}=$ substrat de culture contenant $90 \%$ de terre $+10 \%$ de fumier.
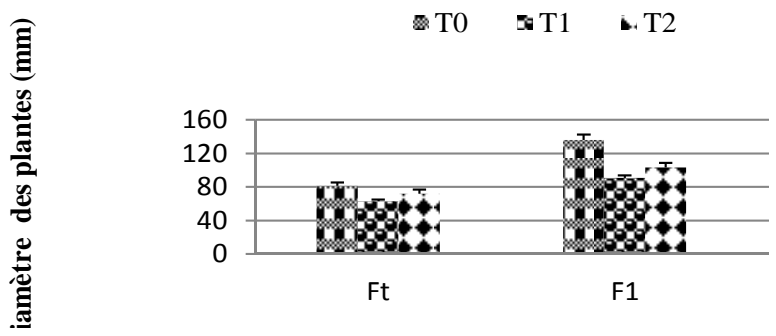

$\mathrm{Ft}$

$\mathrm{F} 1$

Figure 5: Diamètre au collet des plantes d'UAE 22 cultivées sur les trois substrats. T0 = plantes témoins $; \mathbf{T 1}=$ plantes stressées au stade végétatif $; \mathbf{T} 2=$ plantes stressées au stade floraison $; \mathbf{F t}=$ substrat de culture sans fumier $(100 \%$ de terre) $; \mathbf{F 1}=$ substrat de culture composé de $95 \%$ de terre $+5 \%$ de fumier $; \mathbf{F 2}=$ substrat de culture contenant $90 \%$ de terre $+10 \%$ de fumier.
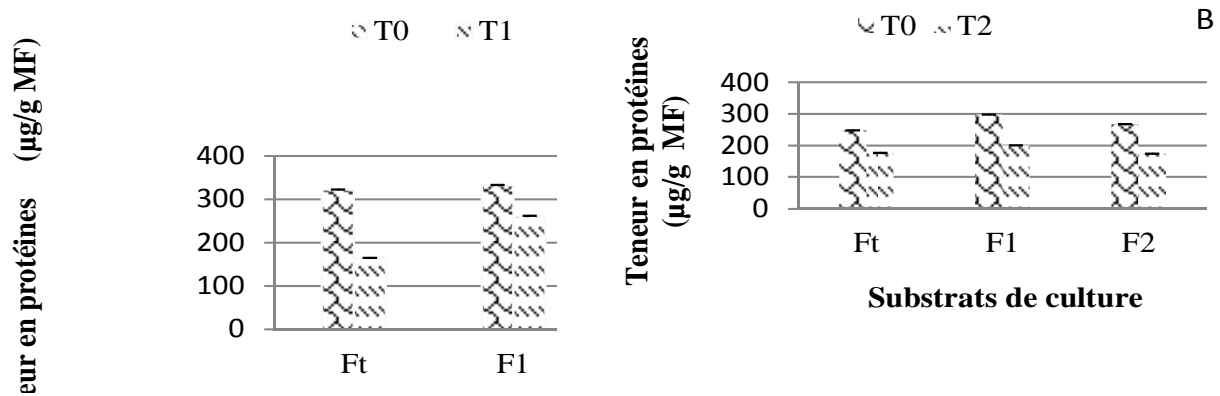

Figure 1: Variation de la teneur en protéines totales des feuilles des plantes d'UAE 22 stressées au stade végétatif $(\mathrm{A})$ et au stade floraison $(\mathrm{B})$, cultivées sur les trois substrats. T0 = plantes témoins ; $\mathbf{T 1}=$ plantes stressées au stade végétatif $; \mathbf{T 2}=$ plantes stressées au stade floraison $; \mathbf{F t}=$ substrat de culture sans fumier $(100 \%$ de terre $) ; \mathbf{F 1}$ $=$ substrat de culture composé de $95 \%$ de terre $+5 \%$ de fumier $; \mathbf{F} 2=$ substrat de culture contenant $90 \%$ de terre $+10 \%$ de fumier.

\section{DISCUSSION}

Selon Ney (2006), l'état hydrique du sol apparaît comme un indicateur du déficit hydrique subi par la plante. La teneur en eau du sol élevée du substrat F2 ainsi que la teneur en eau des feuilles (TRE) élevée des plantes cultivées sur ce même substrat montre que plus il y a du fumier, plus la teneur en eau du sol et la TRE augmentent tandis que l'évapotranspiration diminue. On pourrait expliquer ce phénomène par les faits suivants: le substrat F2 contient moins d'argile et plus 
de potassium ( Tableau 1). Le prélèvement du potassium sous forme d'ions $\mathrm{K}^{+}$confère aux plantes une augmentation de la résistance à la sécheresse; par son effet sur les cellules de garde des stomates il freine la transpiration, maintient la turgescence de la cellule et la régulation de l'économie en eau de la plante. Ces conclusions sont en conformité avec les travaux de Diallo (2009), qui a montré que le fumier (matière organique) procure une meilleure résistance du riz au stress hydrique. Par conséquent Il est considéré comme un facteur externe de résistance au stress hydrique.

La diminution des hauteurs et des diamètres finaux due au stress hydrique constatée, est beaucoup plus accentuée pendant la période végétative parce que le stress a commencé à un stade juvénile ( $32^{\text {ème }}$ jas) et a duré deux semaines, raison pour laquelle les plantes n'ont pas pu rattraper le retard par rapport à celles stressées durant une semaine pendant la floraison. Cependant, les plantes du substrat $\mathrm{F} 1$ enregistrent les meilleurs résultats durant tous les traitements hydriques par rapport aux témoins parce qu'ils se trouvent sur un sol riche en matières organiques. Dans la même logique Diallo et al. (2010) affirment que la hauteur des plants de riz augmente avec la fumure organique à cause de la nutrition azotée qui s'améliore. Le développement des plantes du substrat F1 est accéléré par rapport à celui des plantes du substrat F2 qui sont pourtant cultivées sur un substrat plus riche en matières organiques, en azote, en carbone etc. L'explication de ces résultats, découle du fait que le rapport $\mathrm{C} / \mathrm{N}$ du substrat F2 $(11,47)$ est plus élevé que celui du substrat F1 $(11,04))$. Hors plus $\mathrm{C} / \mathrm{N}$ est élevé, moins l'azote est rapidement disponible. En plus, les plantes du substrat F2 ont eu un début de croissance lent à cause de la salinité du milieu et d'un pH élevé $(8,38)$ suite à un fort pourcentage de fumier contre un $\mathrm{pH}$ de 7,91 pour le substrat F1. A cet effet, Jobin et Petit (2004) affirment que les fumiers et composts sont de bons amendements qui contribuent à maintenir l'humus du sol, mais que les quantités sont souvent trop élevées par rapport aux besoins des légumes.

Il faut rappeler que la restriction hydrique par arrêt d'arrosage a provoqué une baisse de la teneur en protéines totales chez les plantes stressées durant les deux phases de développement. La réduction des taux des composés protéiques chez les plantes en conditions de stress par rapport aux plantes témoins pourrait s'expliquer par la perte de la biomasse. Cette biomasse est aussi constituée de substances organiques et sa perte entraînerait une réduction du taux des composés. La faible teneur en protéines peut être aussi attribuée à la réduction de la surface foliaire et/ou à la fermeture des stomates due au déficit hydrique minimisant les pertes d'eau et provoquant une diminution du rendement à cause de la réduction de la capacité photosynthétique. Ces résultats concordent avec ceux d'autres auteurs tels que, Flexas et Medrano (2002) qui affirment que la fermeture des stomates, pourrait avoir pour conséquence une diminution de la conductance à la diffusion du $\mathrm{CO}_{2}$ et une limitation biochimique du chloroplaste à fixer le $\mathrm{CO}_{2}$. Selon Lowlor (2002), lors d'un déficit hydrique, l'activité physiologique de la feuille, et plus particulièrement la photosynthèse et la conductance stomatique sont affectées. On peut conclure que la baisse de la teneur en protéines totales dans les feuilles en période sèche découle d'une résistance de la plante au stress hydrique. Les plus faibles teneurs en protéines enregistrées chez les plantes cultivées sur le substrat F2 contenant plus de fumier résulteraient de la non disponibilité de l'azote mentionnée plus haut. La forte réduction de la teneur en protéines observée en phase de floraison s'explique par le fait que dans les conditions de développement normale, la période de floraison est marquée par un ralentissement de la croissance parce qu'il y a l'apparition des boutons floraux, l'entretien de la floraison et la fructification (Sawadogo, 2006).

Du point de vue agronomique, le stress hydrique intervenu au stade végétatif ainsi 
qu'au stade de floraison, a provoqué une réduction du rendement en capsules, du poids sec moyen d'une capsule, de la biomasse sèche totale des plantes des substrats de culture F1 et F2 (Tableau5). Ces résultats sont confirmés par ceux de certains auteurs qui ont affirmé que le manque d'eau est un élément déterminant pour la croissance des plantes. Il induit chez les plantes stressées une diminution du contenu relatif en eau, et une réduction significative de la production de biomasse totale (Albouchi et al., 2000), concomitante à une réduction de la croissance en diamètre et en hauteur des tiges (Thomas et Gausling, 2000). Par ailleurs, les valeurs les plus élevées sont enregistrées chez les plantes du substrat F1 qui contient une dose acceptable de fumier, confirmant encore une fois de plus les atouts que possède ce milieu de culture pour un bon développement de la plante et aussi à s'adapter dans des conditions de sécheresse. A cet effet Diallo et al. (2007) affirment que la fumure permet de maintenir des niveaux de production plus élevés par rapport aux témoins par conséquent, contribue à améliorer les rendements en cas de stress modéré et même sévère. Quant au substrat sans fumier Ft, les plantes ont enregistré une augmentation relative du nombre de capsules sous l'effet du stress durant les deux phases de développement. Ces résultats sont en accord avec ceux de Sawadogo et al. (2006) ; Nana (2010) qui ont travaillé sur la variété UAE 22 de gombo et ont souligné que cette variété manifeste une meilleure tolérance au déficit hydrique; en témoigne les meilleurs indices de récolte. Toutefois, l'augmentation est perçue chez les plantes du substrat témoin et non chez les autres, parce qu'elles ont accusé un retard de développement et au moment d'appliquer le stress au stade florifère, elles ne portaient que des boutons floraux pendant que les autres avaient des fleurs ouvertes. Par conséquent, c'est après le stress qu'elles ont pu exprimer leur floraison et le stress a induit une accélération, mais cette production de capsules reste toujours faible par rapport à celle des plantes cultivées sur F1 et F2. La fumure organique contribue une fois de plus à améliorer les rendements des plantes.

L'existence des corrélations positives très significatives entre les paramètres de croissance et agronomiques chez les plantes témoins et leurs absences chez les plantes stressées aux deux stades de développement implique que chez les plantes témoins, la hauteur, le diamètre, la partie végétative aérienne, la partie racinaire souterraine sont étroitement liés et interagissent les uns sur les autres pour le développement harmonieux de la plante tout en influençant le rendement en capsules. Un dysfonctionnement d'un de ces paramètres (conséquence du stress) pourrait affecter négativement le comportement aussi bien morphologique que physiologique de la plante entière. Pour appuyer cette affirmation, Son (2010) stipule que la diminution de rendement observée avec l'augmentation du stress hydrique chez le sésame s'explique surtout par la diminution concomitante au niveau de la taille des plantes, du nombre de ramifications, du nombre et du poids des capsules. Des résultats similaires ont été observés par Messaoudi et El Fellah (2002) sur la vigne; Varasoot et al. (2003) sur l'arachide.

\section{Conclusion}

Au terme de ce travail dont l'objectif était de connaître l'impact du fumier organique sur la réponse du gombo au déficit hydrique, on peut conclure que le stress hydrique a provoqué une réduction de la croissance verticale et radiale des plantes, mais aussi une baisse de la teneur en protéines; la fumure organique a contribué à améliorer significativement la résistance au stress hydrique du gombo. Les plantes stressées cultivées sur les substrats de culture $\mathrm{F} 1$ et $\mathrm{F} 2$ qui ont reçu du fumier ont vu leur TRE, leur croissance verticale et radiale améliorées par rapport à celles stressées cultivées sur le substrat sans fumier. Malgré la hausse du nombre de capsules chez les plantes stressées du substrat sans fumier, ces valeurs restent en deçà de celles des substrats 
amendés. Des deux substrats de culture, le substrat F1 s'est montré plus propice quant à l'amélioration des différents paramètres des plantes de gombo en situation de déficit hydrique. C'est donc le dosage $95 \%$ de terre + $5 \%$ de fumier qui est le mieux adapté pour une culture de gombo dans des conditions hydriques déficitaires.

\section{INTERETS COMPETITIFS}

Les auteurs déclarent qu'ils n'ont aucun conflit d'intérêt.

\section{LES CONTRIBUTIONS DES AUTEURS}

SM de l'Equipe de Génétique et Amélioration des Plantes nous a fournies les semences de gombo pour nos expérimentations. Il a aussi apporté des corrections et suggestions sur le manuscrit et ainsi participé à l'obtention de la version finale du document.

BB et NL de l'Equipe d'Ecophysiologie Végétale ont participé aux semis et aux analyses biochimiques réalisées au laboratoire. NR de l'Equipe d'Ecophysiologie Végétale nous a canalisés dans les expérimentations sur le terrain de bout en bout, nous a aidés avec les analyses statistiques et nous a guidés dans la rédaction $\mathrm{du}$ document. TZ de l'Equipe d'Ecophysiologie végétale, le Directeur de recherche nous a guidé dans le choix du thème d'étude, dans l'élaboration du protocole de recherche. Il a supervisé les travaux, apporté des corrections au manuscrit et ainsi participé à l'obtention de la version finale du document. KB de l'Equipe d'Equipe d'Ecophysiologie Végétale est responsable de tous les travaux de l'expérimentation à la rédaction en passant par les différentes analyses pour l'obtention du DEA.

\section{REFERENCES}

AADI. 2006. Activité de renforcement de commercialisation agricole en Guinée : la filière gombo (okra) en Guinée. PCE-I00-99-00003-00, Task Order N²9, 32p.
Albouchi A, Sebeï A, Mezni MY, El Aouni MA. 2000. Influence de ladurée d'une alimentation déficiente sur la production de biomasse, la surface transpirante et la densité stomatique d'Acacia cyanophylla. Annales de l'INRAGREF, 4: 138-61.

Barrs AD,Weatherley PE. 1968. A reexamination of the relative turgidity technique for estimating water deficits in leaves. Aust. J. Biol. Sci., 15: 413-428.

Bradfort MM. 1976. A rapid and Sensitive Method for the Quantitation of Microgram Quantities of Protein Utilizing the Principle of Protein-Dye Binding. Anal. Biochem., 72: 248-254 .

Diallo D, Tamini Z, Diallo SB, Kourouma D, Barry B, Barry AJ, Diabaye B. 2007. Détermination de la date de semisde quatre variétés de riz en fonction de leur résistance au stress hydrique de fin d'hivernage en vue d'une double culture à Faranah. AGROVISION, 006: 9-16.

Diallo D, Tamini Z, Barry B,Faya AO. 2010. Effet de la fumure organique sur la croissance et le rendement du riz Nerica (WAB 450 IBP 28HB) à Faranah. International Journal of Biological and Chemical Sciences, 4(6): 2017-2025.

Flexas J, Medrano H. 2002. Drought inhibition of photosynthesis in C3 plant:Stomatal and non stomatal limitations revisited. Annals of Botany, 89: 183-189.

Jobin PJ, Petit. 2004. Les amendements organiques: fumiers et composts. 53 pages.

Koulibaly F, Traoré H, Tiendrebéogo L. 2002. Le riz au Burkina Faso, commercialisation, consommation, recherche. Eureka, pp. 77.

Lowlor DW. 2002. Limitation to photosynthesis to water stressed leaves: stomatalvs metabolism and the role of ATP. Annals of Botany, 89: 871-885.

MAE-CIRAD. 2002. Mémonto de l'Agronome. Editions Quae : 1691p.

Messaoudi Z, El Fellah, 2002. Besoin en eau et effet d'un stress hydrique modéré sur la 
croissance et la production de la vigne dans la région de Meknès. A.T. E., 123: 52-55.

Nana R. 2010. Evaluation de la réponse au stress hydrique de cinq variétés de gombo [Abelmoschus esculentus (L.) Moench]. Thèse de Doctorat de l'Universitéde Ouagadougou, 144p.

Ney B. 2006. Analyse et modélisation du peuplement végétal cultivé. In L'Agronomie Aujourd'hui, Dore T, Le Bail M, Martin P, Ney B, Roger-Estrade J (eds). Ed. Cenagref. Cird. Ifremer, I.N.R.A : 99-130.

Oyen LPN, Jemmens RHMJ. 2002. Ressources végétales de l'Afrique tropicale. PROTA précurseur.

Sawadogo M, Balma D, Zombré G. 2006. Expression de différents écotypes de gombo (Abelmoschus esculentus L.) au déficit hydrique intervenu pendant la boutonnisation et la floraison. Base, Biotechnol. Agon. Soc. Environ., 10: 4354.

Sawadogo M, Balma D, Nana R,Sumda RMT. 2009. Diversité agromorphologique et commercialisation du gombo
(Abelmoschus esculentus L.) à Ouagadougou et ses environs. Int. J. Biol. Chem. Sci., 3: 326-336.

Siemonsma J.S, Kouamé C. 2004. Vegetable. Plant Resource of Tropical Africa 2.

Son D. 2010. Effet du stress hydrique sur croissance et le rendement du sésame (Sesamum indicum L.). DEA de l'Université Polytechnique de Bobo Dioulasso, 52p.

Thomas FM, Gausling T. 2000. Morphological and physiological responses of oak seedlings (Quercuspetraces and Q. robur) to moderate drought. Ann. For. Sci.,57: 32533.

VerasootN, Songsri P, Akkasaeng C, Jogloy S, Patanothai A. 2003. Effect of water stress on yield and agronomic chracters of peanut (Arachis hypogea L.). Songklanakarin J. Sci. Technol. 25(3): 283-288. 